\section{Evidence That F Plasmid Transfer Replication Underlies Apparent Adaptive Mutation}

\author{
Timothy Galitski and John R. Roth
}

An Escherichia coli K12 strain, FC40, has been used extensively in the analysis of adaptive mutability. This strain carries a revertible mutant lac allele on an F plasmid and accumulates $\mathrm{Lac}^{+}$(lactose utilizing) revertants, but not unselected mutants, when placed on selective medium. These adaptive mutations are a subset of spontaneous types and their formation depends on the RecABC functions. Data presented here suggest that this phenomenon depends on transfer functions of the $F$ factor. Fertility inhibition eliminates RecA-dependent adaptive reversion. Thus, "adaptive" revertants may form during replication from the transfer origin, whereas loci in the nonreplicating chromosome show little mutation.
The phenomenon variously known as adaptive or directed mutation has provoked considerable controversy and experimentation [reviewed in $(1,2)$ ]. The term adaptive mutation is associated with recent challenges to the neo-Darwinian tenet that all mutations occur spontaneously without regard to fitness. Luria and Delbrück, and the Lederbergs showed that some mutations occur prior to selection (3). The possibility of selectiondirected mutations does not contradict these landmark demonstrations $(4,5)$.

The most complete body of evidence in support of adaptive mutation has involved Escherichia coli mutants defective in lactose utilization. In this system, during extended incubation on minimal lactose medium, the appearance of $\mathrm{Lac}^{+}$revertant colonies is variance $(5,6)$. Each plated cell has a small $\mathrm{Lac}^{+}$. The postplating reversion events occur in a nongrowing cell population $(6,7)$. Mutations at unselected sites do not accumulate (or are not fixed) with time $(5,7)$. Thus, mutability appears to be directed to the base pairs that limit growth. Some workers have proposed alternative interpretations of the observations $(2,8)$. Others have investigated the mechanistic basis of the phenomenon. The latter approach has revealed that postplating reversion depends on homologous recombination functions (RecABC) $(6,9)$. Furthermore, the revertant types that arise during selection are a subset of the types that arise during unselected growth (10). All of these results have been obtained using a single $E$. coli strain, FC40, which carries a mutant lac locus on an F plasmid (6). Here, we present data indicating that the apparent adaptive mutation seen in this strain is dependent on the $F$ plasmid location of the reverting mutation.

Department of Biology, University of Utah, Salt Lake City, UT 84112, USA.

*To whom correspondence should be addressed. linear with time and distributed with a low time-dependent probability of reverting to
The F plasmid belongs to a class of conjugative plasmids that control their sexual functions with a fertility inhibition (Fin) system in which a trans-acting factor, FinO, and antisense RNAs, FinP, combine to repress the expression of an activator, TraJ, of the tra operon (11). The tra operon encodes functions required for conjugal replication and transfer (12). The finO gene of the original F plasmid is interrupted by an IS3 insertion, resulting in constitutive tra operon expression in E. coli K12 (13). However, the conjugal activity of the $\mathrm{F}$ plasmid can be repressed if FinO is supplied by a second, vegetatively compatible plasmid. We have employed the $60 \mathrm{Mda}$ virulence plasmid of Salmonella, pSLT, to provide FinO (14). Salmonella strains with pSLT $\left(\mathrm{Fin}^{+}\right)$show very low mating ability. Removal of the pSLT plasmid stimulates $F$ plasmid transfer 100 - to 1000 -fold (15). By comparing $S$. typhimurium strains that possess or lack pSLT, we have tested the effect of fertility inhibition (Fin) on postplating reversion of the $\mathrm{F}$ plasmid-borne lac mutation.

The F'lac plasmid of strain FC40 was introduced into $\mathrm{Fin}^{+}$and Fin ${ }^{-}$Salmonella strains. This plasmid, $\mathrm{F}^{\prime}$ pro ${ }^{+}$lacl33 3 lacZ, encodes a Lacl-LacZ protein fusion $\left(\mathrm{Lac}^{+}\right.$) with a +1 frameshift in the lacI region $\left(\mathrm{Lac}^{-}\right)$(6). A recA null mutation was introduced into each strain to produce four strains with all combinations of Fin and RecA phenotypes (Table $1 ; 16)$. These strains were plated on a minimal lactose medium with a 50-fold excess of $\mathrm{Lac}^{-}$scavenger cells that were added to consume any other residual carbon sources present in the medium or produced by $\mathrm{Lac}^{+}$revertant colonies. Plates were scored daily for the appearance of new $\mathrm{Lac}^{+}$colonies.

Figure 1A compares the $\mathrm{Lac}^{+}$reversion of $\mathrm{Rec}^{+}$strains TT18302 $\left(\mathrm{Fin}^{+}\right)$and TT18303 (Fin ${ }^{-}$). Both strains produced the same number of spontaneous $\mathrm{Lac}^{+}$revertants (colonies present on day 2). However, the fertile ( Fin $^{-}$) strain accumulated many more revertants after day 2 . On days 3 to 7 , the inhibited $\left(\mathrm{Fin}^{+}\right.$) strain showed a revertant deficit that averaged 78-fold. The same results were obtained when the FinO function was provided by a minimal finO $^{+}$ plasmid (17). Thus, the large pSLT plasmid represses adaptive mutability strictly through inhibition of tra operon expression. Figure 1B shows the $\mathrm{Lac}^{+}$reversion of RecA $^{-}$strains TT18306 (Fin $\left.{ }^{+}\right)$and TT18307 $\left(\right.$ Fin $\left.^{-}\right)$; note the change in scale of the ordinate between Fig. 1A and 1B. The reversion of the fertile $\left(\mathrm{Fin}^{-}\right)$strain was reduced 16 -fold by the recA null mutation (compare Fig. 1A and 1B), whereas the reversion of the inhibited $\left(\mathrm{Fin}^{+}\right)$strain was unaffected by the recA mutation $(P=0.9)$.

Growth of the four tester strains used in the reversion experiments was monitored by the removal of agar plugs from the nonrevertant background, suspension of the cells in saline, and assaying their numbers on appropriate plates (Fig. 2). Salmonella strains such as the scavenger, TR6625, doubled two or three times on the first day and then grew no more; thus, our lactose plates did not support growth (after day 1) of strains without an exogenous lac operon. All strains bearing plasmid $\mathrm{F}^{\prime}$ lacl $33 \Omega$ lac $Z$ showed slow exponential growth throughout a week of incubation. This growth of the $\mathrm{Lac}^{-}$population can account for the exponential accumulation of $\mathrm{Lac}^{+}$revertants (Fig. 1). The $\mathrm{Rec}^{+}$strains grew at a rate of 1.2 doublings per day, whereas the
Table 1. Bacterial strains.

\begin{tabular}{|c|c|}
\hline Strain & Genotype \\
\hline $\begin{array}{l}\text { TT18302 } \\
\text { TT18303 } \\
\text { TT18306 } \\
\text { TT18307 } \\
\text { TR6625 } \\
\text { FC40 }\end{array}$ & 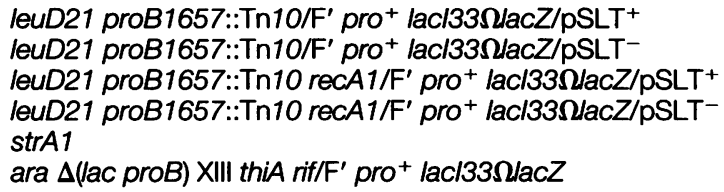 \\
\hline
\end{tabular}

*The four strains TT18302, TT18303, TT18306, and TT18307 are derivatives of strains PM155 (leuD21 pSLT+) and TW101 [leuD21 pSLT ${ }^{-}$; provided by Eisenstadt (26)]. A pro::Tn10 insertion was transduced into these strains and the

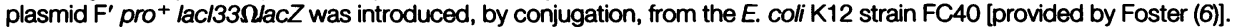
RecA-deficient (recA1) derivatives of these strains were constructed by first introducing an srt:: $\operatorname{Tn} 10 \mathrm{~d} \mathrm{C}$ m mutation; the recA1 mutation was cotransduced into these strains selecting an $\mathrm{Srl}^{+}$phenotype. The Salmonella recA1 allele causes recombination deficiency, UV sensitivity, and a lack of SOS induction (27). All strains, except $E$. coli strain FC40, are derivatives of Salmonella typhimurium LT2. They are naturally $\mathrm{Lac}^{-}$and do not mutate to $\mathrm{Lac}^{+}$without the $\mathrm{F}^{\prime} / \mathrm{ac}$ plasmid. 
$\mathrm{Rec}^{-}$strains grew at 0.88 doublings per day. Note that strains showing very different numbers of $\mathrm{Lac}^{+}$revertants had approximately the same number of $\mathrm{Lac}^{-}$cells on the plate on each day of the experiment (Figs. 1 and 2).

The slow growth imparted to Salmonella strains by the $\mathrm{F}^{\prime}$ lacI33 $\mathrm{Slac} Z$ plasmid differs from the absence of growth of $E$. coli strain FC40 (7), which exhibits linear reversion kinetics $(6,18)$. The mutant $F^{\prime}$ lac produces about the same residual level of $\beta$-galactosidase ( 2 units) in both E. coli and S. typhimurium (19). An absolute lack of growth however, is not necessarily a requirement or a characteristic of adaptive mutation. It is a condition that merely facilitates the observation of apparent adaptive mutability.

Fertility inhibition causes a 78-fold reduction in postplating reversion. The abundant $\mathrm{Lac}^{+}$reversion events of fertile (Fin ${ }^{-}$) strains are dependent on $\operatorname{Rec} A$ function; the residual reversion rate in inhibited $\left(\mathrm{Fin}^{+}\right)$strains is unaffected by a recA null mutation. This associates the Rec-dependent, postplating $\mathrm{Lac}^{+}$reversion of $\mathrm{FC} 40$ with transfer functions of the $F^{\prime}$ lac plasmid. The involvement of plasmid transfer replication in adaptive mutation is supported by independent work (20). We suggest that during incubation on selective medium, cell division and chromosomal replication are prevented or greatly reduced whereas the $\mathrm{F}$ plasmid, carrying the selected locus, is replicating extensively using its sexual origin, oriT. Unselected control loci (which do not accumulate mutations during selection for $\mathrm{Lac}^{+}$) are on the chromosome and are not subject to this private, F-dependent replication and mutability. This difference in location, and not the ability of cells to direct mutability to selectively valuable sites, may account for the apparent directedness of $\mathrm{Lac}^{+}$reversion.

Experiments with strain FC40 have shown that directed reversion occurs only in the presence of lactose and all needed auxotrophic supplements $(6,18)$. Residual $\beta$-galactosidase produced by the mutant lac locus may provide the energy needed by the donor strain for conjugation under starvation conditions (21). Under conditions that limit growth, the $\mathrm{F}$ factor may behave as a selfish genetic element, replicating and moving to new cells. Because host genes are occasionally mobilized, such behavior could have important consequences for the evolution of bacterial populations under commonly encountered stressful conditions. Furthermore, these ideas suggest that $\mathrm{F}$ plasmid fertility functions may be regulated in more complex ways than presently understood.

The spectrum of $\mathrm{Lac}^{+}$revertant types of strain FC40 differs under selective and nonselective conditions (10). Rapidly growing, unselected cultures produce -1 frameshifts (correcting the polar +1 frameshift in the lacl region), and small rearrangements (duplications, deletions) that also relieve polar effects on lacZ. On selective medium the rearrangement class is underrepresented. Small rearrangements like these have been linked to discontinuous lagging-strand DNA synthesis (22). The different mutation spectra may be associated with different modes of DNA replication of the F plasmid [re-
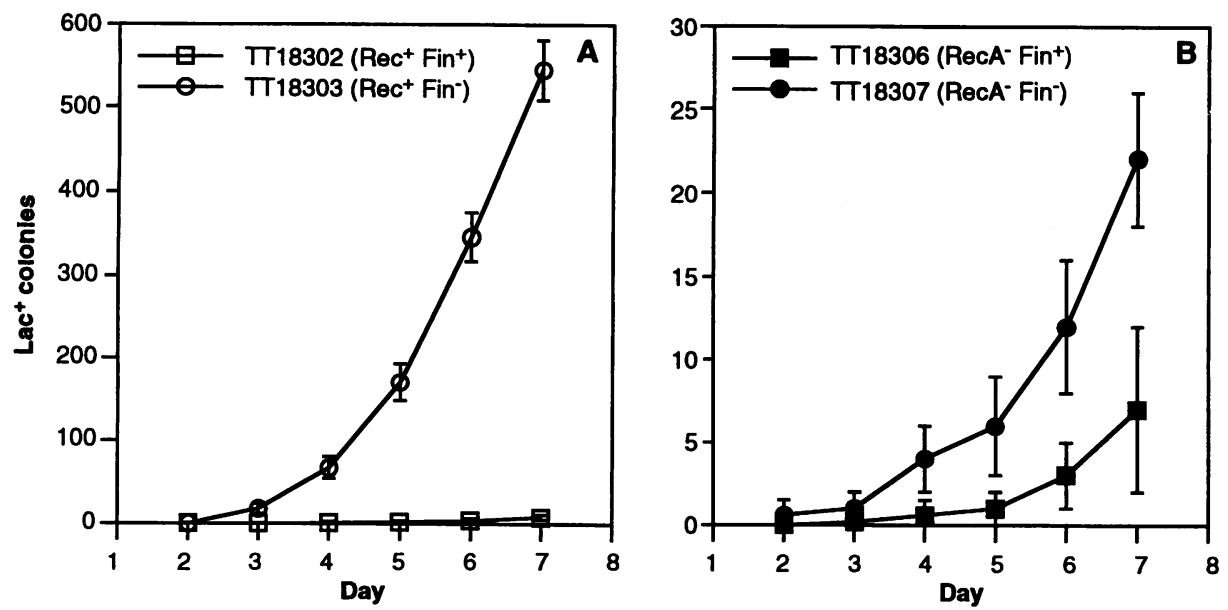

Fig. 1. Effect of fertility inhibition (Fin) and recombination (RecA) functions on $\mathrm{Lac}^{+}$reversion of strains with $\mathrm{F}^{\prime}$ pro ${ }^{+} / a c / 33 \Omega / a c Z$. Cultures of test strains and the scavenger strain, TR6625, were grown to full density in NCE (28) glycerol leucine medium. Cultures were pelleted and resuspended in an equal volume of saline. About $6 \times 10^{6}$ tester cells, with a 50-fold excess of washed scavenger cells, were spread on each selective plate [(A) Rec ${ }^{+}$strains, (B) RecA ${ }^{-}$strains]. Selective medium was NCE $0.2 \%$ lactose Xgal leucine. Leucine was included as an essential nutrient for these auxotrophic strains; it does not provide a carbon or energy source. Plates were incubated for 7 days at $37^{\circ} \mathrm{C}$ in loosely closed plastic sleeves to minimize drying. One plate was designated for daily counting of new $\mathrm{Lac}^{+}$revertant colonies. The others were used to monitor growth of the $\mathrm{Lac}^{-}$population (see Fig. 2). Each point represents the mean and standard deviation of five independent determinations. viewed in (23)]. Under nonselective conditions, the vegetative replication origin, oriV, is the site of initiation of cell cycle-coupled replication with concerted leading- and lagging-strand synthesis. The sexual mode (utilized on selective plates) involves rollingcircle replication initiated at a specific nick at the transfer origin, oriT. Leading-strand replication occurs in the donor cell and replaces a transferred single strand. Lagging-strand replication and Rec-independent plasmid recircularization occur in the recipient cell [reviewed in (24)]. This uncoupled leading- and lagging-strand synthesis may be less prone to generation of small rearrangements.

Our experiments indicate that homologous recombination is required for the observation of mutations generated during transfer replication. In standard conjugation experiments, $F^{\prime}$ plasmid transfer to $\overline{-}$ recipient is independent of $\operatorname{Rec} A$ function in both the donor and the recipient. This may not be true under all conditions. WQ suggest that during growth limitation, trans fer replication (internal or between siblings) seldom goes to completion. This reฮิ sults in linear fragments that cannot circu인 larize but may include a revertant allele these fragments must be incorporated intøo an intact replicon by recombination to al: low formation of a revertant colony.

Though the apparent adaptive mutabil ity in the F'lac situation may be the results of plasmid transfer replication, the possi

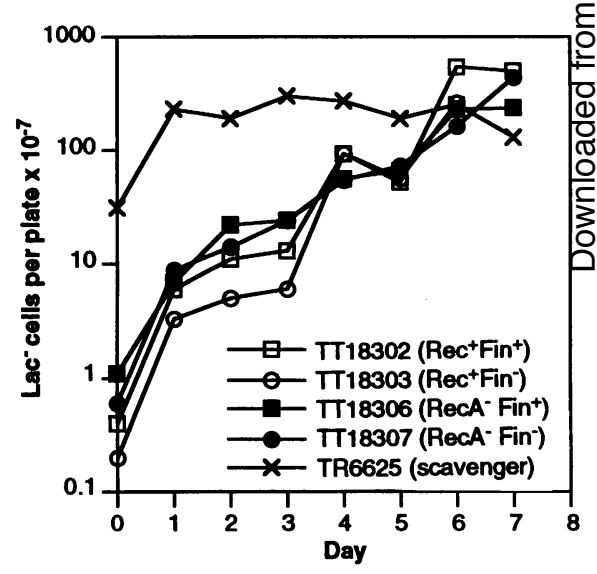

Fig. 2. Growth of mutant cells on minimal lactose medium. From platings described in Fig. 1, agar plugs were taken avoiding $\mathrm{Lac}^{+}$colonies. Bacteria on agar plugs were suspended in saline by vigorous vortex-mixing for $15 \mathrm{~s}$ followed by $15 \mathrm{~min}$ of benchtop incubation without agitation and another 15-s vortex-mixing. Dilutions were prepared in saline and were spread on nutrient agar plates with tetracycline (without tetracycline for assaying scavenger cells alone) to determine the number of viable $\mathrm{Lac}^{-}$tester cells per plate. Shown are the means of five (one for TR6625) independent determinations. Error bars were omitted for clarity. No data were obtained for strain TT18303 on day 7 as a result of the high density of $\mathrm{Lac}^{+}$colonies. 
bility of selection-directed mutation is not eliminated. Adaptive mutation has been investigated in other systems that are not subject to the interpretations proposed here (25). Organisms sense their environment and make directed changes in many aspects of their metabolism. It would seem to be advantageous to direct mutability. We expect that organisms have exploited this possibility.

\section{REFERENCES AND NOTES}

1. P. L. Foster, in Organism and the Origins of Self, A. I. Tauber, Ed. (Kluwer, Norwell, MA, 1991), pp. 213234; J. Bacteriol. 174, 1711 (1992); B. G. Hall, Curr. Opin. Genet. Dev. 2, 943 (1992); F. W. Stahl, Genetics 132, 865 (1992); P. L. Foster, Annu. Rev. Microbiol. 47, 467 (1993)

2. R. E. Lenski and J. E. Mittler, Science 259, 188 (1993).

3. S. E. Luria and M. Delbrück, Genetics 28, 491 (1943); J. Lederberg and E. M. Lederberg, J. Bacteriol. 63, 399 (1952).

4. J. A. Shapiro, Mol. Gen. Genet. 194, 79 (1984).

5. J. Cairns, J. Overbaugh, S. Miller, Nature 335, 142 (1988).

6. J. Cairns and P. L. Foster, Genetics 128, 695 (1991).

7. P. L. Foster, ibid. 138, 253 (1994).

8. B. D. Davis, Proc. Natl. Acad. Sci. U.S.A. 86, 5005 (1989); R. E. Lenski, M. Slatkin, F. J. Ayala, ibid., p. 2775; D. G. MacPhee, Mutat. Res. 285, 109 (1993); J. E. Mittler and R. E. Lenski, Nature 344, 173 (1990); ibid. 356, 446 (1992).

9. R. S. Harris, S. Longerich, S. M. Rosenberg, Science 264, 258 (1994).

10. S. M. Rosenberg, S. Longerich, P. Gee, R. S. Harris, ibid. 265, 405 (1994); P. L. Foster and J. M. Trimarchi, ibid., p. 407.

11. W. B. Dempsey, in Bacterial Conjugation, D. B. Clewell, Ed. (Plenum, New York, 1993), pp. 53-70.

12. L. S. Frost, K. Ippen-Ihler, R. A. Skurray, Microbiol. Rev. 58, 162 (1994).

13. K.-C. Cheah and R. A. Skurray, J. Gen. Microbiol 132, 3269 (1986)

14. M. J. Gasson and N. S. Willetts, J. Bacteriol. 122 518 (1975).

15. K. E. Sanderson, S. K. Kadam, P. R. MacLachlan, Can. J. Microbiol. 29, 1205 (1983).

16. To confirm the Fin phenotypes, strains were assayed for donor proficiency using the pro $^{+}$gene present on the F'lac plasmid. The Fin ${ }^{-}$strains TT18303 (Rec $^{+}$ Fin $\left.^{-}\right)$and TT18307 (RecA- Fin $\left.{ }^{-}\right)$have donor proficiencies (Pro ${ }^{+}$transconjugants per donor cell) of 0.34 and 0.60 , respectively. The donor proficiencies of their Fin ${ }^{+}$counterparts, TT18302 and TT18306, are 100 - to 200 -fold lower.

17. To strain $\mathrm{TT}_{18303}\left(\mathrm{rec}^{+} \mathrm{pSLT}^{-}\right)$we introduced plasmid pWD51, a minimal finO ${ }^{+}$pBR322-based plasmid clone kindly provided by W. Dempsey [S. A. Mclntire and W. B. Dempsey, Nucleic Acids Res. 15, 2029 (1987)]. This derivative behaved like strain TT18302 ( $\left.\mathrm{rec}^{+} \mathrm{pSLT}^{+}\right)$in tests of donor proficiency (16) and adaptive reversion (Fig. 1A). A pBR322 plasmid vector control had no effect.

18. P. L. Foster and J. Cairns, Genetics 131, 783 (1992)

19. The residual $\beta$-galactosidase activities (encoded by

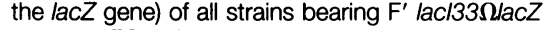
including FC40 (Table 1), were assayed. All had activities of about 2 Miller units. Assays were performed as described except that the growth medium was NCE (28) $0.2 \%$ glycerol with leucine; J. H. Miller, Experiments in Molecular Genetics (Cold Spring Harbor Laboratory, Cold Spring Harbor, NY, 1972).

20. J. E. Peters and S. A. Benson, J. Bacteriol. 177, 847 (1995); J. P. Radicella, P. U. Park, M. S. Fox, Science 268, 418 (1995).

21. K. W. Fisher, J. Gen. Microbiol. 16, 136 (1957).

22. T. Q. Trinh and R. R. Sinden, Nature 352, 544 (1991).

23. N. Willetts and R. Skurray, in Escherichia coli and Salmonella typhimurium: Cellular and Molecular Biology, F. C. Neidhardt, Ed. (American Society for Microbiology, Washington, DC, 1987), pp. 1110-1133.
24. B. Wilkins and E. Lanka, in Bacterial Conjugation, D. B. Clewell, Ed. (Plenum, New York, 1993), pp. 105-136.

25. B. A. Bridges, Mutation Res. 307, 149 (1994); G Maenhaut-Michel and J. A. Shapiro, EMBO J. 13, 5229 (1994); P. L. Foster and J. Cairns, ibid., p. 5240; B. G. Hall, Mol. Biol. Evol. 11, 159 (1994); J. Bacteriol. 175, 6411 (1993); D. F. Steele and S. Jinks-Robertson, Genetics 132, 9 (1992); B. G. Hall, Proc. Natl. Acad. Sci. U.S.A. 89, 4300 (1992).

26. PM155 from: K. M. Overbye and P. Margolin, J. Bacteriol. 146, 170 (1981); TW101 from: W. H Koch, T. A. Cebula, P. L. Foster, E. Eisenstadt, ibid. 174, 2809 (1992).
27. J. P. Wing, M. Levine, H. O. Smith, ibid. 95, 1828 (1968); T. Galitski, unpublished data.

28. D. Berkowitz, J. M. Hushon, H. J. J. Whitfield, J. Roth, B. N. Ames, J. Bacteriol. 96, 215 (1968)

29. The authors thank M. Fox, J. P. Radicella, P. Park, J. Cairns, P. Foster, J. Trimarchi, S. Rosenberg, R. Harris, E. Eisenstadt, W. Dempsey, F. Stahl, and N. $P$. Higgins for sharing unpublished data, strains, and helpful discussions. This work was supported by grant GM27068 (J.R.R.) and predoctoral training grant GM07464-15 (T.G.) from the National Institutes of Health.

9 February 1995; accepted 23 March 1995

\section{Cloning of the $\beta$ Cell High-Affinity Sulfonylurea Receptor: A Regulator of Insulin Secretion}

\section{Lydia Aguilar-Bryan, ${ }^{*}$ Colin G. Nichols, Sérgio W. Wechsler, John P. Clement IV, A. E. Boyd III,† Gabriela González, Haydée Herrera-Sosa, Kimberly Nguy, Joseph Bryan, $\ddagger$ Daniel A. Nelson $\ddagger \S$}

Sulfonylureas are a class of drugs widely used to promote insulin secretion in the treatment of non-insulin-dependent diabetes mellitus. These drugs interact with the sulfonylurea receptor of pancreatic $\beta$ cells and inhibit the conductance of adenosine triphosphate (ATP)-dependent potassium $\left(\mathrm{K}_{\mathrm{ATP}}\right)$ channels. Cloning of complementary DNAs for the high-affinity sulfonylurea receptor indicates that it is a member of the ATP-binding cassette or traffic ATPase superfamily with multiple membrane-spanning domains and two nucleotide binding folds. The results suggest that the sulfonylurea receptor may sense changes in ATP and ADP concentration, affect $\mathrm{K}_{\text {ATP }}$ channel activity, and thereby modulate insulin release.

Sulfonylureas are oral hypoglycemics widely used in the treatment of non-insulindependent diabetes mellitus to stimulate insulin release from pancreatic islet $\beta$ cells. The mechanism of stimulation is through inhibition of an ATP-dependent potassium channel, $K_{A T P}$, which sets the $\beta$ cell resting membrane potential (1). A reduction of potassium outflow causes $\beta$ cell depolarization and the activation of one or more L-type calcium channels (2). The resulting calcium influx triggers exocytosis (3). Sulfonylureas like tolbutamide or glyburide decrease $\mathrm{K}_{\mathrm{ATP}}$ channel activity, which depolarizes the cell and prompts insulin release.

The $K_{A T P}$ channels and sulfonylurea receptors (SURs) appear to be functionally linked, although it is not clear if they are a

L. Aguilar-Bryan, S. W. Wechsler, J. P. Clement IV, A. E. Boyd III, G. González, H. Herrera-Sosa, K. Nguy, J. Bryan, D. A. Nelson, Departments of Cell Biology and Medicine, Baylor College of Medicine, Houston, TX 77030, USA.

C. G. Nichols, Department of Cell Biology and Physiology, Washington University School of Medicine, St. Louis, MO 63110, USA

*To whom correspondence should be addressed. tDeceased. This author contributed to the initiation of this work while Head of the Division of Endocrinology at Baylor College of Medicine.

$\ddagger$ These authors contributed equally to this work.

§Present address: Department of Biology, University of North Carolina, Charlotte, NC 28223, USA. single entity (4-6). Biochemical studies indicate that SUR is a large membrane protein (140 to $170 \mathrm{kD}$ ) (7) that can bind sulfonylureas with high affinity (dissociation constant $K_{\mathrm{d}}<10 \mathrm{nM}$ ) (1). The affinity of sulfonylureas for SUR is decreased by nucleotides and possibly phosphorylation (8). It has been proposed that the $K_{\text {ATP }}$ channel contains a sulfonylurea binding domain, a binding site for potassium channel openers, and two or more nucleotide binding sites that can discriminate between ATP and ADP (9). One report has placed the nucleotide binding sites and the sulfonylurea binding site on the same large molecule with an ATP affinity label (10).

Glyburide (11) and an iodinated derivative of glyburide (12) can be cross-linked by photolabeling to a protein with an apparent molecular size of $140 \mathrm{kD}$. The pharmacological characteristics of the photolabeling (12), the low-nanomolar $K_{\mathrm{d}}$, and the appropriate rank order displacement with other insulin-releasing sulfonylureas are expected from studies on glyburide-induced insulin release and inhibition of $K_{A T P}$ channel activity. These results indicate that the $140-\mathrm{kD}$ molecule is SUR. We used a radioiodinated derivative of glyburide (12) to purify hamster SUR (13) and obtain the $\mathrm{NH}_{2}$-terminal amino acid sequence from 